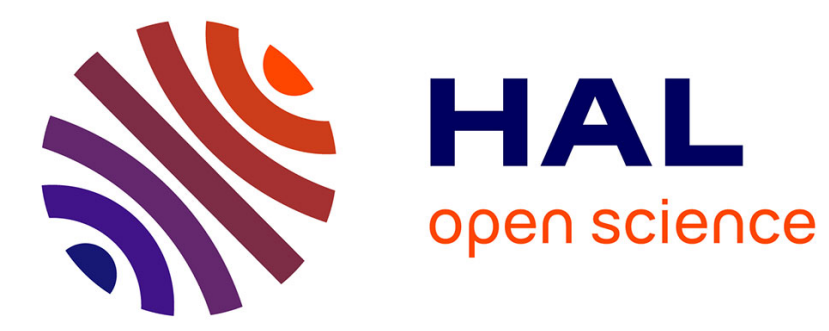

\title{
SPEED : Mining Maxirnal Sequential Patterns over Data Strearns
}

Chedy Raïssi, Pascal Poncelet, Maguelonne Teisseire

\section{To cite this version:}

Chedy Raïssi, Pascal Poncelet, Maguelonne Teisseire. SPEED: Mining Maxirnal Sequential Patterns over Data Strearns. IS: Intelligent Systems, Sep 2006, London, United Kingdom. pp.546-552, 10.1109/IS.2006.348478. hal-00134387

\section{HAL Id: hal-00134387 https://hal.science/hal-00134387}

Submitted on 1 Mar 2007

HAL is a multi-disciplinary open access archive for the deposit and dissemination of scientific research documents, whether they are published or not. The documents may come from teaching and research institutions in France or abroad, or from public or private research centers.
L'archive ouverte pluridisciplinaire HAL, est destinée au dépôt et à la diffusion de documents scientifiques de niveau recherche, publiés ou non, émanant des établissements d'enseignement et de recherche français ou étrangers, des laboratoires publics ou privés. 


\section{SPEED : Mining Maximal Sequential Patterns over Data Streams}

\author{
Chedy Raïssi \\ EMA-LGI2P/Site EERIE \\ Parc Scientifique Georges \\ Besse \\ 30035 Nîmes Cedex, France
}

\author{
Pascal Poncelet ${ }^{\dagger}$ \\ EMA-LGI2P/Site EERIE \\ Parc Scientifique Georges \\ Besse \\ 30035 Nîmes Cedex, France
}

\author{
Maguelonne Teisseire \\ LIRMM UMR CNRS 5506 \\ 161 Rue Ada, 34392 \\ Montpellier cedex 5 - France
}

\begin{abstract}
Many recent real-world applications, such as network traffic monitoring, intrusion detection systems, sensor network data analysis, click stream mining and dynamic tracing of financial transactions, call for studying a new kind of data. Called stream data, this model is, in fact, a continuous, potentially infinite flow of information as opposed to finite, statically stored data sets extensively studied by researchers of the data mining community. An important application is to mine data streams for interesting patterns or anomalies as they happen. For data stream applications, the volume of data is usually too huge to be stored on permanent devices, main memory or to be scanned thoroughly more than once. We thus need to introduce approximations when executing queries and performing mining tasks over rapid data streams. In this paper we propose a new approach, called Speed (Sequential Patterns Efficient Extraction in Data streams), to identify frequent maximal sequential patterns in a data stream. To the best of our knowledge this is the first approach defined for mining sequential patterns in streaming data. The main originality of our mining method is that we use a novel data structure to maintain frequent sequential patterns coupled with a fast pruning strategy. At any time, users can issue requests for frequent maximal sequences over an arbitrary time interval. Furthermore, our approach produces an approximate support answer with an assurance that it will not bypass a user-defined frequency error threshold. Finally the proposed method is analyzed by a series of experiments on different datasets.
\end{abstract}

\section{INTRODUCTION}

Recently, the data mining community has focused on a new challenging model where data arrives sequentially in the form of continuous rapid streams. It is often referred to as

\footnotetext{
*email: Chedy.Raissi@ema.fr

$\dagger$ email: Pascal.Poncelet@ema.fr

‡email: teisseire@lirmm.fr
}

data streams or streaming data. Since data streams are continuous, high-speed and unbounded, it is impossible to mine association rules by using algorithms that require multiple scans. As a consequence new approaches were proposed to maintain itemsets $[7,4,3,6,13]$. Nevertheless, according to the definition of itemsets, they consider that there is no limitation on items order. In this paper we consider that items are really ordered into the streams, therefore we are interested in mining sequences rather than itemsets. We propose a new approach, called SpeEd (Sequential Patterns Efficient Extraction in Data streams), to mine sequential patterns in a data stream. The main originality of our approach is that we use a novel data structure to incrementally maintain frequent sequential patterns (with the help of tilted-time windows) coupled with a fast pruning strategy. At any time, users can issue requests for frequent sequences over an arbitrary time interval. Furthermore, our approach produces an approximate support answer with an assurance that it will not bypass a user-defined frequency thresholds.

The remainder of the paper is organized as follows. Section 2 goes deeper into presenting the problem statement. In Section 3 we propose a brief overview of related work. The SPEED approach is presented in Section 4. Section 5 reports the result of our experiments. In Section 6, we conclude the paper.

\section{PROBLEM STATEMENT}

The traditional sequence mining problem was firstly introduced in [12] and extended in [11] as follows. Let $D B$ be a set of customer transactions where each transaction $T$ consists of customer-id, transaction time and a set of items involved in the transaction. Let $I=\left\{i_{1}, i_{2} \ldots i_{m}\right\}$ be a set of literals called items. An itemset is a non-empty set of items. A sequence $s$ is a set of itemsets ordered according to their timestamp. It is denoted by $\left\langle s_{1} s_{2} \ldots s_{n}\right\rangle$, where $s_{j}, j \in 1 \ldots n$, is an itemset. A $k$-sequence is a sequence of $k$ items (or of length $k$ ). A sequence $S^{\prime}=<s_{1}^{\prime} s_{2}^{\prime} \ldots s_{n}^{\prime}>$ is a subsequence of another sequence $S=<s_{1} s_{2} \ldots s_{m}>$, denoted $S^{\prime} \prec S$, if there exist integers $i_{1}<i_{2}<\ldots i_{j} \ldots<i_{n}$ such that $s_{1}^{\prime} \subseteq s_{i_{1}}, s_{2}^{\prime} \subseteq s i_{2}, \ldots s_{n}^{\prime} \subseteq s i_{n}$.

All transactions from the same customer are grouped together and sorted in increasing order and are called a data sequence. A support value (denoted $\operatorname{support}(S)$ ) for a sequence gives its number of actual occurrences in $D B$. Nevertheless, a sequence in a data sequence is taken into account only once to compute the support even if several occurrences are discovered. A data sequence contains a sequence $S$ if $S$ 
is a subsequence of the data sequence. In order to decide whether a sequence is frequent or not, a minimum support value, denoted $\sigma$, is specified by the user, and the sequence is said to be frequent if the condition :

$$
\operatorname{support}(S) \geq \sigma \text { holds. }
$$

The anti-monotonic Apriori property [1] holds for sequential patterns [10].

Given a database of customer transactions the problem of sequential pattern mining is to find all the sequences whose support is greater than a specified threshold (minimum support). Each of these represents a sequential pattern, also called a frequent sequence. The task of discovering all the frequent sequences is quite challenging since the search space is extremely large: let $\left\langle\begin{array}{llll}s_{1} & s_{2} & \ldots & s_{m}\end{array}\right\rangle$ be a provided sequence and $n_{i}=\left|s_{j}\right|$ cardinality of an itemset. Then the search space, i.e. the set of all potentially frequent sequences is $2^{n_{1}+n_{2}+\ldots n_{m}}$.

Let us now examine the problem when considering streaming data. Let data stream $D S=B_{a_{i}}^{b_{i}}, B_{a_{i+1}}^{b_{i+1}}, \ldots, B_{a_{n}}^{b_{n}}$ be an infinite sequence of batches, where each batch is associated with a time period $\left[a_{k}, b_{k}\right]$, i.e. $B_{a_{k}}^{b_{k}}$ with $b_{k}>a_{k}$, and let $B_{a_{n}}^{b_{n}}$ be the most recent batch. Each batch $B_{a_{k}}^{b_{k}}$ consists of a set of customer data sequences; that is, $B_{a_{k}}^{b_{k}}=\left[S_{1}, S_{2}\right.$, $\left.S_{3}, \ldots, S_{j}\right]$. For each data sequence $S$ in $B_{a_{k}}^{b_{k}}$ we are thus provided with its list of itemsets. In the rest of the paper we will consider, without loss of generality, that an itemset is merely reduced to one item. We also assume that batches do not have necessarily the same size. Hence, the length $(L)$ of the data stream is defined as $L=\left|B_{a_{i}}^{b_{i}}\right|+\left|B_{a_{i+1}}^{b_{i+1}}\right|+\ldots+\left|B_{a_{n}}^{b_{n}}\right|$ where $\left|B_{a_{k}}^{b_{k}}\right|$ stands for the cardinality of the set $B_{a_{k}}^{b_{k}}$. In this

\begin{tabular}{|c|c|c|}
\hline$B_{0}^{1}$ & $\begin{array}{l}S_{a} \\
S_{b} \\
\end{array}$ & $\begin{array}{l}(1)(2)(3)(4)(5) \\
(8)(9)\end{array}$ \\
\hline$B_{1}^{2}$ & $\overline{S_{c}}$ &  \\
\hline$B_{2}^{3}$ & $\begin{array}{l}S_{d} \\
S_{e} \\
S_{f}\end{array}$ & $\begin{array}{l}(1)(2)(3) \\
(1)(2)(8)(9) \\
(2)(1)\end{array}$ \\
\hline
\end{tabular}

Figure 1: The set of batches $B_{0}^{1}, B_{1}^{2}$ and $B_{2}^{3}$

context, we define the support of a sequential pattern as follows: the support of a sequence $S$ at a specific time interval $\left[a_{i}, b_{i}\right]$ is denoted by the ratio of the number of customers having sequence $S$ in the current time window to the total number of customers. Therefore, given a user-defined minimum support, the problem of sequential patterns in data streams is to find all frequent patterns $S_{k}$ over an arbitrary time period $\left[a_{i}, b_{i}\right]$, i.e. verifying :

$$
\sum_{t=a_{i}}^{b_{i}} \operatorname{support}_{t}\left(S_{k}\right) \geq \sigma \times\left|B_{a_{i}}^{b_{i}}\right|
$$

of the streaming data using as little main memory as possible.
EXAMPLE 1. In the rest of the paper we will use this toy example as an illustration, while assuming that the first batch $B_{0}^{1}$ is merely reduced to two customer data sequences. Figure 1 illustrates the set of all batches. Let us now consider the following batch, $B_{1}^{2}$, which only contains one customer data sequence. Finally we will also assume that three customer data sequences are embedded in $B_{2}^{3}$. Let us now assume that the minimum support value is set to $50 \%$. If we look at $B_{0}^{1}$, we obtain the two following maximal frequent patterns: $<(1)(2)(3)(4)(5)>$ and $\langle(8)(9)>$. If we now consider the time interval $[0-2]$, i.e. batches $B_{0}^{1}$ and $B_{1}^{2}$, maximal frequent patterns are: $\langle(1)(2)\rangle$. Finally when processing all batches, i.e. a $[0-3]$ time interval, we obtain the following set of frequent patterns: $\langle(1)(2)\rangle,\langle(1)\rangle$ and $\langle(2)\rangle$. According to this example, one can notice that the support of the sequences can vary greatly depending on the time periods and so it is highly needed to have a framework that enables us to store these time-sensitive supports.

\section{RELATED WORK}

In the recent years, data streams mining approaches mainly focused on maintaining frequent itemsets over the entire history of a streaming data. The first approach was proposed by Rajeev and Motwani [7] where they study the landmark model where patterns support is calculated from the start of the data stream. The authors also define the first singlepass algorithm for data streams based on the anti-monotonic property. Li et al. [6] use an extended prefix-tree-based representation and a top-down frequent itemset discovery scheme. Chi et al. [3] consider closed frequent itemsets and propose the closed enumeration tree (CET) to maintain a dynamically selected set of itemsets.

In [4], authors consider a FP-tree-based algorithm [5] to mine frequent itemsets at multiple time granularities by a novel logarithmic tilted-time windows tables technique. Figure 2 shows a natural tilted-time windows table: the most recent 4 quarters of an hour, then, in another level of granularity, the last 24 hours, and 31 days. Based on this model, one can store and compute data in the last hour with the precision of quarter of an hour, the last day with the precision of hour, and so on. By matching for each sequence of a batch a tilted-time window, we have the flexibility to mine a variety of frequent patterns depending on different time intervals. In [4], the authors propose to extend natural tilted-time windows table to logarithmic tilted-time windows table by simply using a logarithmic time scale as shown in Figure 3. The main advantage is that with one year of data and a finest precision of quarter, this model needs only 17 units of time instead of 35,136 units for the natural model. In order to maintain these tables, the logarithmic tilted-time windows frame will be constructed using different levels of granularity each of them containing a userdefined number of windows. Let $B_{1}^{2}, B_{2}^{3}, \ldots, B_{n-1}^{n}$ be an infinite sequence of batches where $B_{1}^{2}$ is the oldest batch. For $i \geq j$, and for a given sequence $S$, let $\operatorname{Support}_{i}^{j}(S)$ denote the support of $S$ in $B_{i}^{j}$ where $B_{i}^{j}=\bigcup_{k=i}^{j} B_{k}$. By using a logarithmic tilted-time windows table, the following supports of $S$ are kept: Support $_{n-1}^{n}(S) ;$ Support $_{n-2}^{n-1}(S) ;$ Support $_{n-4}^{n-2}(S)$; Support $_{n-6}^{n-4}(S) \ldots$. This table is updated as follows. Given a new batch B, we first replace $\operatorname{Support}_{n-1}^{n}(S)$, the frequency at the finest level of time granularity (level 0 ), with Support $(B)$ and shift back to the next finest level of time 
granularity (level 1). Support ${ }_{n-1}^{n}(S)$ replaces Support $_{n-2}^{n-1}(S)$ at level 1. Before shifting Support $_{n-2}^{n-1}(S)$ back to level 2, we check if the intermediate window for level 1 is full (in this example the maximum windows for each level is 2). If yes, then Support $t_{n-2}^{n-1}(S)+$ Support $_{n-1}^{n}(S)$ is shifted back to level 2. Otherwise it is placed in the intermediate window and the algorithm stops. The process continues until shifting stops. If we received $N$ batches from the stream, the logarithmic tilted-time windows table size will be bounded by $2 \times\left\lceil\log _{2}(N)\right\rceil+2$ which makes this windows schema very space-efficient.



Figure 2: Natural Tilted-Time Windows Table

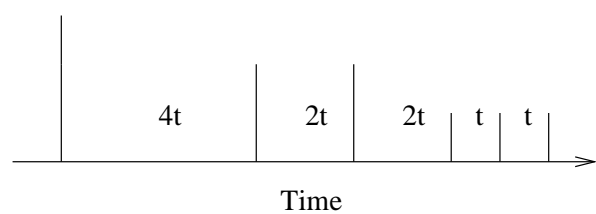

Figure 3: Logarithmic Tilted-Time Windows Table

According to our problem, all presented approaches consider inter-transaction associations, i.e. there is no limitation on order of events while we consider sequences, which implies a strict order of events. By considering such an order, we are thus provided with a challenging problem since the search space is significantly larger. In [2], authors also address sequential patterns mining however they focus on patterns across multiple data streams. They consider as input a set of streams, i.e. time series composed of categorial values as in [9], while we are interested in patterns occurring in an unique and continuous data stream.

\section{THE SPEED APPROACH}

In this section we propose the SPEED approach for mining sequential patterns in streaming data.

\subsection{An overview}

Our main goal is to mine all maximal sequential patterns over an arbitrary time interval of the stream. The algorithm runs in 2 steps:

1. The insertion of each sequence of the studied batch in the data structure Lattice $_{\text {reg }}$ using the region principle.

2. The extraction of the maximal subsequences.

We will now focus on how each new batch is processed then we will have a closer look on the pruning of unfrequent sequences.

From the batches from Example 1, our algorithm performs as follows: we process the first sequence $S_{a}$ in $B_{0}^{1}$ by first

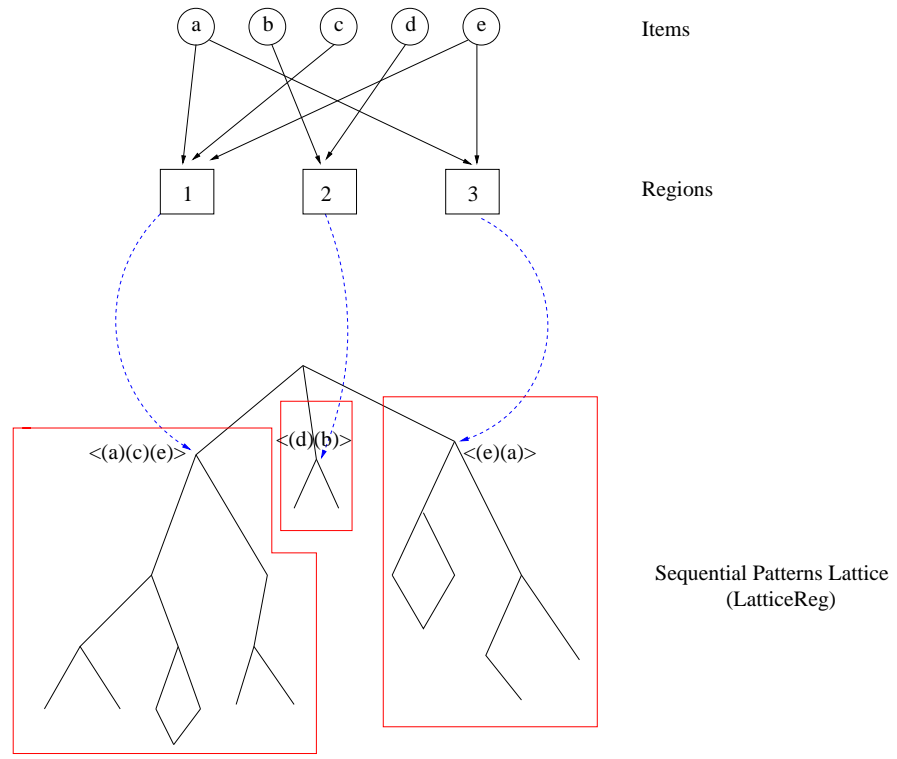

Figure 4: The data structures used in SPEED algorithm

\begin{tabular}{|c|c|c|}
\hline Items & Tilted-T W & (Regions, Root $\left._{\text {Reg }}\right)$ \\
\hline \hline 1 & $\left\{\left[t_{0}=1\right]\right\}$ & $\left\{\left(1, S_{a}\right)\right\}$ \\
2 & $\left\{\left[t_{0}=1\right]\right\}$ & $\left\{\left(1, S_{a}\right)\right\}$ \\
3 & $\left\{\left[t_{0}=1\right]\right\}$ & $\left\{\left(1, S_{a}\right)\right\}$ \\
4 & $\left\{\left[t_{0}=1\right]\right\}$ & $\left\{\left(1, S_{a}\right)\right\}$ \\
5 & $\left\{\left[t_{0}=1\right]\right\}$ & $\left\{\left(1, S_{a}\right)\right\}$ \\
\hline
\end{tabular}

Figure 5: Updated items and there support after the sequence $S_{a}$

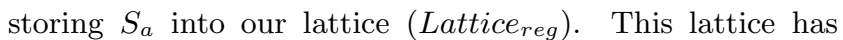
the following characteristics: each path in Lattice $_{r e g}$ is provided with a region and sequences in a path are ordered according to the inclusion property. By construction, all subsequences of a sequence are in the same region. This lattice is used in order to reduce the search space when comparing and pruning sequences. Furthermore, only maximal sequences are stored into Lattice $_{\text {reg. }}$. These sequences are either sequences directly extracted from batches or their maximal subsequences which are constructed from items of a sequence such as all these items are in the same region. Such a merging operation has to respect item order in the sequence, i.e. this order is expressed through their timestamp. By storing only maximal subsequences we aim at storing a minimal number of sequences such that we are able to answer a user query. When the processing of $S_{a}$ completes, we are provided with a set of items (1..5), one sequence $\left(S_{a}\right)$ and Lattice $_{\text {reg }}$ updated. Items are stored as illustrated in Figure 5. The "Tilted-T $W$ " attribute is the number of

\begin{tabular}{|c|c|c|}
\hline Sequences & Size & Tilted-Time Windows \\
\hline \hline$S_{a}$ & 5 & $\left\{\left[t_{0}=1\right]\right\}$ \\
\hline$S_{b}$ & 2 & $\left\{\left[t_{0}=1\right]\right\}$ \\
\hline
\end{tabular}

Figure 6: Updated sequences after the sequence $S_{b}$ 


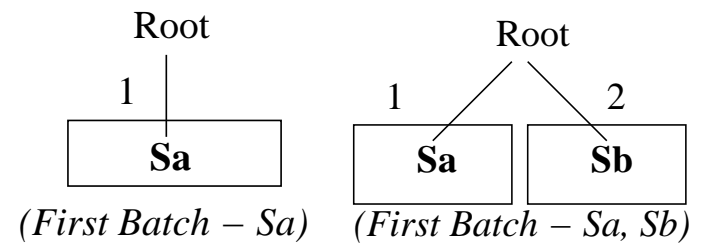

Figure 7: The region lattice after the first batch

occurrences of the corresponding item in the batch. The "Root reg attribute stands for the root of the corresponding region in Lattice $_{\text {reg. }}$ Of course, for one region we only have one Root $_{\text {Reg }}$ and we also can have several regions for one item. For sequences (C.f. Figure 6), we store both the size of the sequence and the associated tilted-time window. This information will be useful during the pruning phase. The left part of the Figure 7 illustrates how the Lattice $_{r e g}$ lattice is updated when considering $S_{a}$.

Let us now process the second sequence of $B_{0}^{1}$. Since $S_{b}$ is not a subsequence of $S_{a}$, it is inserted in Lattice $_{r e g}$ in a new valuation (C.f. subtree $S_{b}$ in Figure 7).

\begin{tabular}{|c|c|c|}
\hline Items & Tilted-T W & (Regions, Root Reg $)$ \\
\hline \hline 1 & $\left\{\left[t_{0}=1\right],\left[t_{1}=1\right]\right\}$ & $\left\{\left(1, S_{a}\right)\right\}$ \\
2 & $\left\{\left[t_{0}=1\right],\left[t_{1}=1\right]\right\}$ & $\left\{\left(1, S_{a}\right)\right\}$ \\
$\ldots$ & $\ldots$ & $\ldots$ \\
8 & $\left\{\left[t_{0}=1\right]\right\}$ & $\left\{\left(2, S_{b}\right)\right\}$ \\
9 & $\left\{\left[t_{0}=1\right]\right\}$ & $\left\{\left(2, S_{b}\right)\right\}$ \\
\hline
\end{tabular}

Figure 8: Updated items after $B_{1}^{2}$

\begin{tabular}{|c|c|c|}
\hline Sequences & Size & Tilted-Time Windows \\
\hline \hline$S_{a}$ & 5 & $\left\{\left[t_{0}=1\right]\right\}$ \\
\hline$S_{c}$ & 2 & $\left\{\left[t_{0}=1\right],\left[t_{1}=1\right],\left[t_{2}=1\right]\right\}$ \\
\hline$S_{d}$ & 3 & $\left\{\left[t_{0}=1\right],\left[t_{2}=1\right]\right\}$ \\
\hline$\ldots$ & $\ldots$ & $\ldots$ \\
\hline
\end{tabular}

Figure 9: Updated sequences after $S_{d}$ of $B_{2}^{3}$

Let us now consider the batch $B_{1}^{2}$ merely reduced to $S_{c}$. Since items 1 and 2 already exist in the set of sequences, their tilted-time windows must be updated (C.f. Figure 8). Furthermore, items 1 and 2 are in the same region: 1 and the longest subsequence for these items is $<(1)(2)>$, i.e. $S_{c}$ which is included in $S_{a}$. We thus have to insert $S_{c}$ in Lattice $_{\text {reg }}$ in the region 1 . Nevertheless as $S_{c}$ is a subsequence of $S_{a}$ that means that when $S_{a}$ occurs in previous batch it also occurs for $S_{c}$. So the tilted-time window of $S_{c}$ has to be also updated.

The sequence $S_{d}$ is considered in the same way as $S_{c}$ (C.f. Figure 10 and Figure 12). Let us now have a closer look on the sequence $S_{e}$. We can notice that items 1 and 2 are in region 1 while items 8 and 9 are in region 2 . We can believe that we are provided with a new region. Nevertheless, we can notice that in fact the sequence $<(8)(9)>$ already exist in Lattice $_{r e g}$ and is a subsequence of $S_{e}$. The longest subsequence of $S_{e}$ in the region 1 is $\langle(1)(2)\rangle$. In the

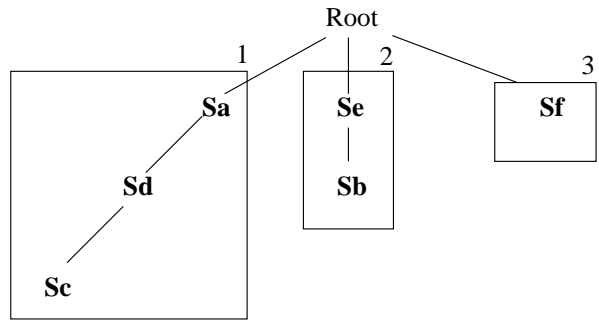

(Third Batch $-\mathrm{Se})$

Figure 10: The region lattice after batches processing

\begin{tabular}{|c|c|c|}
\hline Items & Tilted-T W & $($ Regions, Root Reg $)$ \\
\hline \hline 1 & $\left\{\left[t_{0}=1\right],\left[t_{1}=1\right]\right.$ & $\left\{\left(1, S_{a}\right)\right\}$ \\
&,$\left.\left[t_{2}, 2\right]\right\}$ & $\left\{\left(2, S_{e}\right)\right\}$ \\
& & $\left\{\left(3, S_{f}\right)\right\}$ \\
\hline 2 & $\left\{\left[t_{0}=1\right],\left[t_{1}=1\right]\right.$ & $\left\{\left(1, S_{a}\right)\right\}$ \\
&,$\left.\left[t_{2}, 2\right]\right\}$ & $\left\{\left(2, S_{e}\right)\right\}$ \\
& & $\left\{\left(3, S_{f}\right)\right\}$ \\
\hline$\ldots$ & $\ldots$ & $\ldots$ \\
\hline
\end{tabular}

Figure 11: Updated items after the sequence $S_{f}$

same way, the longest subsequence of $S_{e}$ for region 2 is < $(8)(9)>$. As we are provided with two different regions and $\langle(8)(9)>$ is the root of region 2 , we do not create a new region but we insert $S_{e}$ as a root of region for 2 and we insert the subsequence $\langle(1)(2)\rangle$ both on lattice for region 1 and 2. Of course, tilted-time windows tables are updated. Finally we proceed to the last sequence $S_{f}$. We can notice that the order between itemsets is different from previous sequences. When parsing the set of items, we can conclude that they occur in the same region 1 . Nevertheless the longest subsequences are reduced to $\langle(1)>$ and $<$ (2) > , i.e. neither $S_{f} \prec S_{c}$ or $S_{c} \prec S_{f}$ holds, then we have to consider a new region.

To only store frequent maximal subsequences, let us now discuss how unfrequent sequences are pruned. While pruning in [4] is done in 2 distinct operations, SPEED prunes unfrequent patterns in a single operation which is in fact a dropping of the tail sequences of tilted-time windows $\operatorname{Support}_{k}^{k+1}(S)$, Support $_{k+1}^{k+2}(S), \ldots$, Support $_{n-1}^{n}(S)$ when the following condition holds: $\forall i, k \leq i \leq n$, support $a_{a_{i}}^{b_{i}}(S)<\varepsilon_{f}\left|B_{a_{i}}^{b_{i}}\right|$.

By navigating into Lattice reg, and by using the regions in-

\begin{tabular}{|c|c|c|}
\hline Sequences & Size & Tilted-Time Windows \\
\hline \hline$S_{a}$ & 5 & $\left\{\left[t_{0}=1\right]\right\}$ \\
\hline$S_{b}$ & 2 & $\left\{\left[t_{0}=1\right],\left[t_{2}=1\right]\right\}$ \\
\hline$S_{c}$ & 2 & $\left\{\left[t_{0}=1\right],\left[t_{1}=1\right],\left[t_{2}=2\right]\right\}$ \\
\hline$S_{d}$ & 3 & $\left\{\left[t_{0}=1\right],\left[t_{2}=1\right]\right\}$ \\
\hline$S_{e}$ & 4 & $\left\{\left[t_{2}=1\right]\right\}$ \\
\hline$S_{f}$ & 2 & $\left\{\left[t_{2}=1\right]\right\}$ \\
\hline
\end{tabular}

Figure 12: Updated sequences after $S_{f}$ of $B_{2}^{3}$ 
dex, we can directly and rapidly prune irrelevant sequences without further computations. This process is repeated after each new batch in order to use as little main memory as possible. During the pruning phase, titled-windows are merged in the same way as in [4].

\subsection{The SPeEd algorithm}

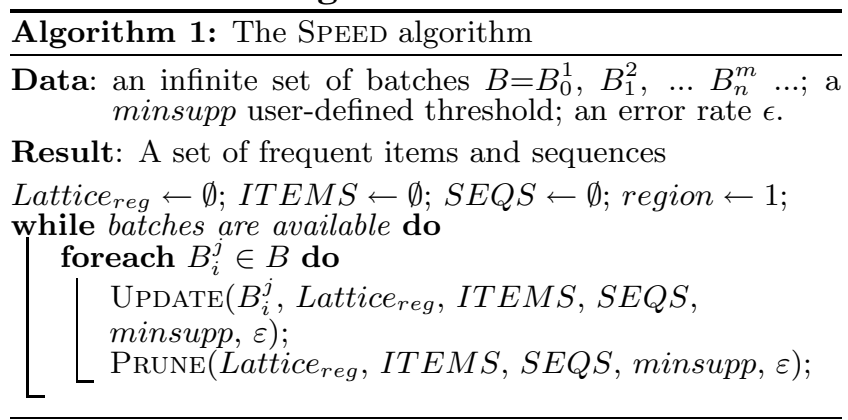

We describe in more detail the SPEED algorithm (C.f. Algorithm 1). While batches are available, we consider sequences embedded in batches in order to update our structures (UPDATE). Then we prune unfrequent sequences in order to maintain our structures in main memory (PRUNETREE). In the following, we consider that we are provided with the three next structures. Each value of ITEMS is a tuple (labelitem, $\left\{\right.$ time $\left._{\text {occ }}\right\},\left\{\left(\right.\right.$ regions Root $\left.\left._{\text {Reg }}\right)\right\}$ ) where labelitem stands for the considered item, $\{$ time, occ $\}$ is used in order to store the number of occurrences of the item for different time of batches and for each region in \{regions we store its associated sequences $\left(\right.$ Root $_{\text {Reg }}$ in the Lattice $_{\text {reg }}$ structure. According to the following property, the number of regions is limited w.r.t the number of items in $D S$.

Property 1. Let $\Phi$ be the number of items in DS. The maximal number of regions is bounded by $\Phi^{2}+1$.

Proof: Let $\Phi$ be the number of items. We can generate $\Phi^{2}$ maximal sequences of size 2 and one maximal sequence. Each of them stands for a region. Whatever the added sequence, it will be a subsequence and will be included in one of the already existing $\Phi^{2}+1$ regions.

In other words, in the worst case, our algorithm has to check, for each sequence embedded in a batch $\Phi^{2}+1$ regions.

The $S E Q S$ structure is used to store sequences. Each value of $S E Q S$ is a tuple $(s$, size $(s),\{$ time, occ $\})$ where size $(s)$ stands for the number of items embedded in $s$. Finally, the Lattice $_{r e g}$ structure is a lattice where each node is a sequence stored in $S E Q S$ and where vertices correspond to the associated region (according to the previous overview) $\square$

Let us now examine the Update algorithm (C.f. Algorithm 2 ) which is the main core of our approach. We consider each sequence embedded in the batch. From a sequence $S$, we first get regions of all its items (GETREGIONs). If items were not already considered we only have to insert $S$ in a new region. Otherwise, we extract all different regions associated on items of $S$. For each region the GetFirstSeqOfVAL function returns a new subsequence $S_{x}$ constructed by merging items sharing same region with their associated Root $_{\text {Reg }}$. We then compute the longest common subsequences of $S_{x}$ in Root $_{V a l}$ by applying the LCSP Longest Common Sequential Patterns function. This function returns an empty set




both when there are no subsequences or if subsequences are merely reduced to one item ${ }^{1}$.

Property 2. Let $u, v$ be two sequences and $|u|,|v|$ the associated size. Let $r$ be the size of the maximal subsequence between $u$ and $v$. Let $\Lambda$ be the number of maximal subsequences. We have: $\Lambda \leq\left(\begin{array}{c}w=\min (|u|,|v|) \\ r\end{array}\right)$.

Proof: Let $u$ and $v$ be two sequences. We can obtain respectively $2^{|u|}$ and $2^{|v|}$ subsequences. The set of maximal subsequences having size $r$ is then: $\min \left(\left(\begin{array}{c}|u| \\ r\end{array}\right),\left(\begin{array}{c}|v| \\ r\end{array}\right)\right) \equiv$ $\left(\begin{array}{c}w=\min (|u|,|v|) \\ r\end{array}\right) \square$

If there is only one subsequence, i.e. cardinality of NewSeq is 1 , we know that the subsequence is either a root of region or $S_{x}$ itself. We thus store it in a temporary array (LatticeMerge). This array will be used in order to avoid to create a new region if it already exists a root of region included in $S$. Otherwise we know that we are provided with a subsequence and then we insert it into Lattice reg $_{\text {(INSERT) and propagate }}$ the tilted-time window (UPDATETTW). Sequences are also stored in a temporary array (DelayedInsert). If there exist more than one subsequence, then we insert all these subsequences on the corresponding region and also store with $S$ on DelayedInsert them in order to delay their insertion for a new region. If LatticeMerge is empty we know that it does not exist any subsequence of $S$ included on sequences of Lattice $_{\text {reg }}$ and then we can directly insert $S$ in a new region. Otherwise, we insert the subsequence in Lattice $_{\text {reg }}$ for the region of LatticeMerge. If the cardinality of LatticeMerge is greater than 1 , we are provided with a sequence which will be a new root of region and then we insert it. For the both last case, we insert all the set of subsequences embedded and we update their tilted-time windows (INSERTANDUPDATEALL).

Property 3. Let Lattice $_{\text {reg }}$ be the structure at the end of the process. Let $S$ and $S^{\prime}$ be two sequences such as $S^{\prime} \preceq S$, then: 1. If $S^{\prime}$ does not exist in Lattice $_{\text {reg }}$ then $S$ also does not exist in Lattice $_{\text {reg. }}$. 2. If $S^{\prime}$ exists in Lattice $_{\text {reg }}$, let Sup ${ }_{0}^{\prime}, \ldots$, Sup $_{n}^{\prime}$ (resp. Sup $p_{0}, \ldots$, Sup $p_{m}$ for $S$ ) be the supports of $S^{\prime 2}$ in all of its tilted-time windows then: $n \geq m$ and Sup $_{i}^{\prime} \geq$ Sup $_{i}, \forall i$ such as $0 \leq i \leq m$

Proof: The first part is proved by induction on N, i.e. the number of batches. For $N=0$ (the oldest batch), if $S \in$ Lattice $_{\text {reg }}$ then Support ${ }_{0}(S) \geq \epsilon_{f}\left|B_{0}^{1}\right|$. Let us consider that $S^{\prime} \notin$ Lattice $_{\text {reg }}$, as $S^{\prime} \nsubseteq S$ we have $\operatorname{Support}_{0}(S) \leq \epsilon_{f}\left|B_{0}^{1}\right|$. So we have $S \notin$ Lattice $_{\text {reg. }}$. For $N>0$, let us consider $S^{\prime} \notin$ Lattice $_{\text {reg }}$ after processing the $N^{\text {th }}$ batch. We have to consider the two following cases: (i) $S^{\prime} \notin$ Lattice $_{\text {reg }}$ after the processing of the $(N-1)^{t h}$ batch then by induction we also have $S \notin$ Lattice $_{\text {reg }}$ by extending in the same way the case $N=0$. (ii) $S^{\prime} \in$ Lattice $_{\text {reg }}$ after the $(N-1)^{t h}$ batch and the sequence was pruned when processing the batch $N$. As $S^{\prime} \nsubseteq S$, it exists $S_{1}, \ldots, S_{p}$ such as $S_{1}=S^{\prime}$ and $S_{p}=S$, where $S_{i}$ is the subsequence of $S_{i+1}$ for $1 \leq i \leq p$. With the pruning condition, we know that $S_{1}, \ldots, S_{p}$ were already pruned during the processing of the batch $N$ thus

\footnotetext{
${ }^{1}$ LCSP is an extension of the NKY algorithm [8] of time complexity $O(n(m-r))$ where $n$ and $m$ are the sizes of sequences and $r$ the size of the longest maximal sequence. ${ }^{2}$ where $S u p_{0}^{\prime}$ is the support in the most recent window.
}

$S \notin$ Lattice $_{\text {reg. }}$.

As $S^{\prime} \leq S$, we have $S_{1}, \ldots, S_{p}$ (cf previous part). By using the pruning condition, we know that the table of tiltedtime windows of $S_{i}$ has much more windows than $S_{i+1}$ with $1 \leq i \leq p$, thus $S^{\prime}$ has much more windows that $S(m \geq n)$. By definition, we know that Support $_{0}, \ldots$, Support $_{n-1}$ and Support $t_{0}^{\prime}, \ldots$, Support ${ }_{n-1}^{\prime}$ are the support of $S$ and $S^{\prime}$ for each batch. These windows have the same structure, we can thus apply the anti-monotonic property: $\operatorname{Support}_{i}\left(S^{\prime}\right) \geq$ $\operatorname{Support}_{i}(S)$ for $1 \leq i \leq n-1$. Let us assume $W_{s}$ (resp. $\left.W_{s^{\prime}}\right)$, the set of sequences having incremented $S$ (resp. $S^{\prime}$ ) for the batch $N$, i.e $\operatorname{Suppport}_{n}(S)=\left|W_{s}\right|$. We thus have $W_{s} \subseteq W_{s^{\prime}}$ and by the antimonotonic property: $\operatorname{Support}_{q}\left(S^{\prime}\right)=$ $\left|W_{s^{\prime}}\right| \geq \operatorname{Support}_{q}(S)=\left|W_{s}\right| \cdot \square$

Maintaining all the data streams in the main memory requires too much space. So we have to store only relevant sequences and drop sequences when the tail-dropping condition holds. When all the tilted-time windows of the sequence are dropped the entire sequence is dropped from Lattice reg. $_{\text {. }}$ As a result of the tail-dropping we no longer have an exact support over $L$, rather an approximate support. Now let us denote $\operatorname{Support}_{L}(S)$ the frequency of the sequence $S$ in all batches and Support $_{L}(S)$ the approximate frequency. With $\varepsilon \ll$ minsupp this approximation is assured to be less than the actual frequency according to the following inequality [4]:

$$
\operatorname{Support}_{L}(S)-\epsilon|L| \leq \operatorname{Support}_{L}(S) \leq \operatorname{Support}_{L}(S) \text {. }
$$

Due to lack of space we do not present the entire PRUNE algorithm we rather explain how it performs. First all sequences verifying the pruning constraint are stored in a temporary set (ToPrune). We then consider items in ITEMS data structure. If an item is unfrequent, then we navigate through Lattice $_{\text {reg }}$ in order:

\section{1. to prune this item in sequences}

2. to prune sequences in Lattice $_{r e g}$ also appearing in ToPrune

This function takes advantage of the anti-monotonic property as well as the order of stored sequences. It performs as follows, nodes in Lattice $_{r e g}$, i.e. sequences, are pruned until a node occurring in the path and having siblings is found. Otherwise, each sequence is updated by pruning the unfrequent item. When an item remains frequent, we only have to prune sequences in ToPrune by navigating into Lattice reg $_{\text {. }}$

\section{EXPERIMENTS}

In this section, we report our experiments results. The stream data was generated by the IBM synthetic marketbasket data generator ${ }^{3}$. In all the experiments we used $1 \mathrm{~K}$ distinct items and generated $1 \mathrm{M}$ of transactions. Furthermore, we have fixed minsupp at $10 \%$. We conducted two sets of experiments, in the first, we set the frequency error threshold at 0.1 with an average sequence length of 3 or 5

\footnotetext{
${ }^{3}$ http://www.almaden.ibm.com/cs/quest.
} 
itemsets and in the second we set the frequency error threshold at 0.2 with the same sequence lengths. The stream was broken in batches of 20 seconds for the 3 -sequences and 90 seconds for the 5-sequences. Furthermore, all the transactions can be fed to our program through standard input. Finally, our algorithm was written in $\mathrm{C}++$ and compiled using gcc without any optimizations flags. All the experiments were performed on an AMD Athlon XP-2200 running Linux with $512 \mathrm{MB}$ of RAM.
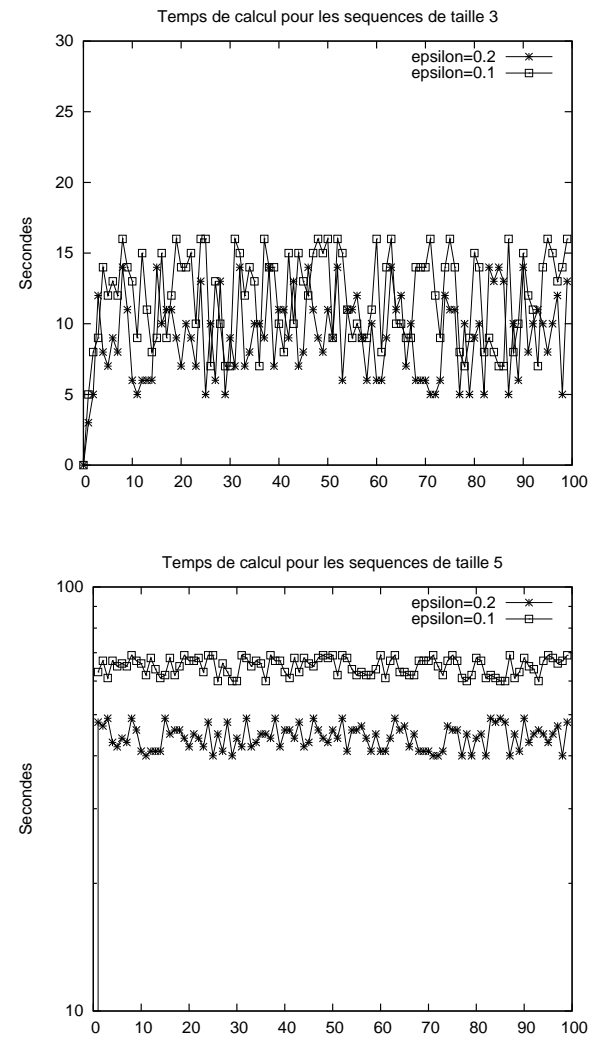

Figure 13: Speed Time requirements for $\{3,5\}-$ sequences

At each processing of a batch the following informations were collected: the size of the SPEED data structure at the end of each batch in bytes, the total number of seconds required per batch, the total number of maximal sequences generated for this batch and the number of valuations present on the data stream sequences. The $\mathrm{x}$ axis represents the batch number. Figure 13 show time results for 3 and 5-sequences. Every two batches the algorithm needs more time to process sequences, this is in fact due to the merge operation of the tilted-time windows which is done in our experiments every 2 batches on the finest granularity level. The jump in the algorithm is thus the result of extra computation cycles needed to merge the tilted-time windows values for all the nodes in the Lattice $_{\text {reg }}$ structure. The time requirements of the algorithm tend to grow very slowly as the stream progresses and do not excess the 20 or the 90 seconds computation time limit for every batch. Figure 14 show memory needs for the processing of our sequences. Space requirements is bounded for 3 -sequences by $35 \mathrm{M}$ and
$78 \mathrm{M}$ for the 5 -sequences, this requirement is however acceptable as this can easily fit in main memory. Experiments show that the SPEED algorithm can handle sequences in data streams without falling behind the stream as long as we choose correct batch duration values.
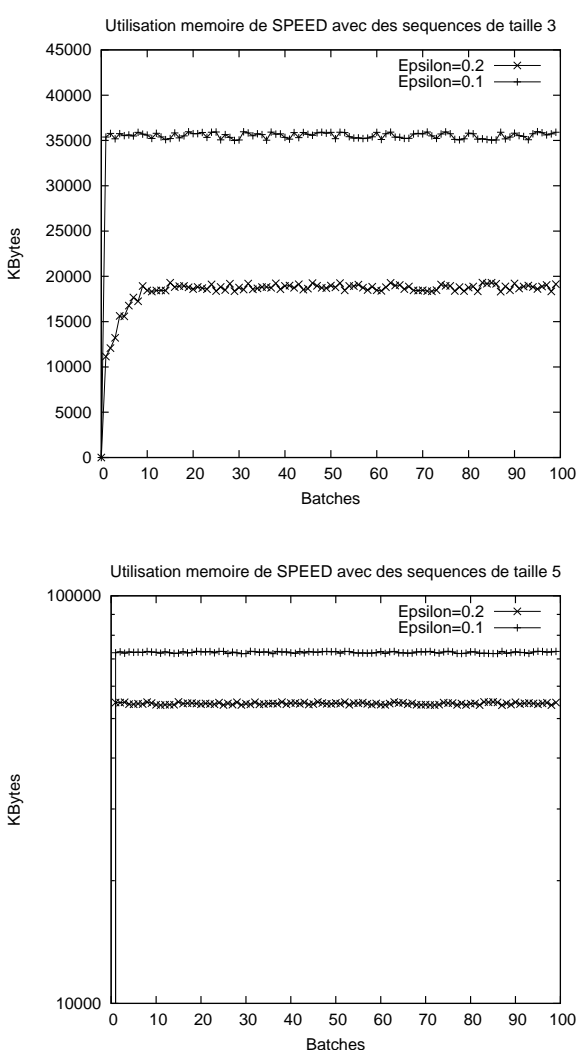

Figure 14: Speed Memory requirements for $\{3-5\}-$ sequences

\section{CONCLUSION}

In this paper we addressed the problem of mining sequential patterns in streaming data and proposed the first approach, called SPEED, for mining such patterns. SPEED is based on a new efficient structure and on strict valuation of edges. Such a valuation is very useful either when considering the pruning phase or when comparing sequences since we only have to consider sequences embedded into the lattice sharing same valuations. Thanks to the anti-monotonic property and the order of stored sequences in our structure, the pruning phase is also improved. Conducted experiments have shown that our approach is efficient for mining sequential patterns in data stream. Furthermore, with SPEED, users can, at any time, issue requests for frequent sequences over an arbitrary time interval.

\section{REFERENCES}

[1] R. Agrawal, T. Imielinski, and A. Swami. Mining association rules between sets of items in large database. In Proceedings of the International Conference on Management of Data (ACM SIGMOD 93), pages 207-216, 1993. 
[2] G. Chen, X. Wu, and X. Zhu. Mining sequential patterns accross data streams. Technical Report CS-05-04, University of Vermont, march 2005.

[3] Y. Chi, H. Wang, P.S. Yu, and R.R. Muntz. Moment: Maintaining closed frequent itemsets over a stream sliding window. In Proceedings of the 4th IEEE International Conference on Data Mining (ICDM 04), pages 59-66, Brighton, UK, 2004.

[4] G. Giannella, J. Han, J. Pei, X. Yan, and P. Yu. Mining frequent patterns in data streams at multiple time granularities. In In H. Kargupta, A. Joshi, K. Sivakumar and Y. Yesha (Eds.), Next Generation Data Mining, MIT Press, 2003.

[5] J. Han, J. Pei, B. Mortazavi-asl, Q. Chen, U. Dayal, and M. Hsu. Freespan: Frequent pattern-projected sequential pattern mining. In Proceedings of the 6th International Conference on Knowledge Discovery and Data Mining (KDD 00), pages 355-359, Boston, USA, 2000 .

[6] H.-F. Li, S.Y. Lee, and M.-K. Shan. An efficient algorithm for mining frequent itemsets over the entire history of data streams. In Proceedings of the 1st International Workshop on Knowledge Discovery in Data Streams, Pisa, Italy, 2004.

[7] G. Manku and R. Motwani. Approximate frequency counts over data streams. In Proceedings of the 28th International Conference on Very Large Data Bases (VLDB 02), pages 346-357, Hong Kong, China, 2002.

[8] Yajima Shuzo Nakatsu Narao, Kambayashi Yahiko. A longest common subsequence suitable for similar text strings. Acta Informatica, 18(1):171-179, 1982.

[9] T. Oates and P. Cohen. Searching for structure in multiple streams of data. In Proceedings of the 13th International Conference on Machine Learning (ICML 96), pages 346-354, Bari, Italy, 1996.

[10] J. Pei, J. Han, and W. Wang. Mining sequential patterns with constraints in large databases. In Proceedings of the 10th International Conference on Information and Knowledge Management (CIKM 02), pages 18-25, MCLean, USA, 2002.

[11] R. Srikant and R. Agrawal. Mining sequential patterns: Generalizations and performance improvements. In Proceedings of the 5th International Conference on Extending Database Technology (EDBT 96), pages 3-17, Avignon, France, 1996.

[12] R. Agrawal R. Srikant. Mining sequential patterns. In Proceedings of the 11th International Conference on Data Engineering (ICDE 95), pages 3-14, Tapei, Taiwan, 1995.

[13] W.-G. Teng, M.-S. Chen, and P.S. Yu. A regression-based temporal patterns mining schema for data streams. In Proceedings of the 29th International Conference on Very Large Data Bases (VLDB 03), pages 93-104, Berlin, Germany, 2003. 